that Caliban's body activates implicit considerations over the competing claims of hereditary owners of the land and its labourers.

The last chapter of the book links early modern England to today's global world in a powerful, acute way. Akhimie shares a personal anecdote about her brother, a big, tall, dark-skinned man, who took up the habit of walking around his neighbourhood in central Florida while she was writing this book. In the wake of the numerous killings of unarmed black men by US law enforcement officers during the so-called "pedestrian checks," the scholar shares her worries about her brother not making it back home after one of those evening walks. By linking another genteel early modern activity such as pedestrianism to today's common habit of walking, Akhimie urges readers to think about the fluidity and adaptability of race as a social construct. She also encourages readers to understand that racism is a historical phenomenon that is pervasive in our everyday lives. If pedestrianism in early modern conduct literature was a healthy activity for the freeborn to enjoy the benefits of the free air, this form of self improvement is not possible for some individuals today as they are seen and checked as potential threats on the basis of their own somatic marks. Bodily marks deviating from the dominant group's somatic norm are still racial signifiers affecting, more or less overtly, people's lives. By connecting past and present notions of race, Akhimie argues for the necessity of a more inclusive rhetoric that could dismantle the naturalizing discourses of racial profiling and discrimination limiting some individuals' ability to succeed.

DANIELE LAUDADIO

University of Toronto

\title{
Alonge, Guillaume. \\ Condottiero, cardinale, eretico. Federico Fregoso nella crisi politica e religiosa del Cinquecento.
}

Studi e testi del Rinascimento europeo, 42. Rome : Edizioni di Storia e Letteratura, 2017. xxiv, 392 p. ISBN 978-8-8935-9015-0 (broché) €44.80.

Ce premier ouvrage de Guillaume Alonge s'inscrit dans un vaste débat historiographique international sur la question de l'évangélisme et de ses ramifications. Depuis les recherches de Delio Cantimori, qui s'est interrogé dans 
les années 1930 sur les courants hétérodoxes du XVI e siècle et dont l'héritage est encore aujourd'hui bien vivant, de multiples travaux sur la Réforme et la dissidence religieuse ont fleuri dans la péninsule italienne. On peut rappeler les études d'Adriano Prosperi, de Benedetto Croce, de Carlo Ginzburg, mais aussi de Massimo Firpo sous la direction duquel Guillaume Alonge a d'ailleurs conduit sa thèse en co-tutelle avec Olivier Christin. Dans l'introduction de son livre, l'auteur ne manque pas de rappeler les travaux de Pierre Imbart de la Tour qui, le premier, a proposé la formule si controversée d' "évangélisme ", ceux de Jonathan A. Reid qui a consacré un travail majeur au réseau évangélique de Marguerite de Navarre et la thèse de Thierry Wanegffelen qui a développé une lecture singulière de la question de ces hommes du "plat pays de la croyance " (xiii). Avec cet ouvrage, G. Alonge interroge à nouveau frais ce mouvement de réforme de l'Église à travers l'étude de la figure de l'évêque génois Federico Fregoso (1480-1541), personnage majeur qui joua un rôle important dans le tissage de liens entre le mouvement de l'évangélisme français et les milieux réformateurs de la péninsule. Cette figure aujourd'hui trop souvent méconnue est brossée à grands traits dans l'introduction (ix-xxi) dans laquelle l'auteur s'emploie à retracer son parcours, son milieu de vie, l'importance des réseaux dans lesquels s'inscrit sa puissante famille et, plus généralement, le contexte politique et religieux si particulier de ce début du XVI siècle. Le lecteur comprend en effet rapidement, dès le premier des neuf chapitres qui composent le volume, combien l'histoire familiale de Fregoso est fondamentale pour saisir son brillant destin. Si le jeune homme, après son exil de Gênes du fait de la mort brutale de son père " condottiero ", occupe rapidement une place à part à la cour d'Urbino, c'est parce qu'il a un lien très étroit avec le pouvoir ducal : il est en effet, par un jeu subtil de mariages croisés, le petit-fils du duc d'Urbino Federico Montefeltro. Grâce à ce dernier, grand mécène et parfaite incarnation de ces princes de la Renaissance qui apprécient et soutiennent l'art et la culture, le jeune Fregoso rencontre les plus grands artistes du temps. Ainsi, il côtoie Raphaël, Castiglione, l'auteur du Courtisan, mais aussi Pietro Bembo, père de la langue italienne. G. Alonge montre d'ailleurs la présence des deux frères Federico et Ottaviano parmi les personnages principaux du Courtisan et explique comment, au fil des réécritures du livre (trois ou quatre fois entre 1509 et 1528), Castiglione fait évoluer les caractères des personnages en fonction de la progression de leur statut social et politique. Un important développement est consacré à ce que l'auteur appelle la "génération du Courtisan " (13-29), 
ces jeunes hommes qui quittent les États de la péninsule et se dirigent vers le centre de la chrétienté, Rome, qui est désormais le seul lieu du pouvoir (avec Venise) encore en capacité de conserver une autonomie et une certaine initiative politique vis-à-vis des grandes monarchies européennes qui, depuis quelques temps déjà, ont transformé l'Italie en une zone de combats et de rivalités.

$\mathrm{Au}$ fil des chapitres qui suivent un ordre chronologique efficace et documenté, le lecteur découvre l'existence sinueuse de Fregoso, cet archevêque guerrier qui n'hésite pas à prendre l'épée en 1516 pour défendre les intérêts politiques et commerciaux de son frère lorsque celui-ci réussit à devenir le doge de leur cité natale (31). Un homme plus intéressé par les questions politiques que religieuses, qui s'allie à François $\mathrm{I}^{\mathrm{er}}$ au détriment de Rome, de Milan et du roi d'Espagne, mais qui bientôt, notamment après la disparition de son frère (1524), traverse une profonde crise spirituelle. À la cour du roi de France et à Lyon, il est alors marqué par trois influences spirituelles majeures : celles de Savonarole, de Luther et de Lefèvre d'Etaples dont il découvre les écrits en côtoyant des membres du cercle évangélique de Marguerite de Navarre. De 1527 à 1529, alors qu'il a été nommé par le roi de France abbé de l'abbaye bénédictine Saint-Bénigne de Dijon, il se laisse habiter par les idées de l'évangélisme français et se convainc de la nécessité de réformer l'institution ecclésiastique. Lorsqu'il retourne dans la péninsule en 1529 et qu'il s'installe dans son évêché de Gubbio (197-244 : «Un evangelismo pastorale»), il se lance dans une sérieuse tentative de réforme: G. Alonge décrit ses efforts pour remettre de l'ordre dans les monastères, rétablir l'autorité de l'évêque tout en publiant de nombreux statuts synodaux. Mais cette fervente activité ne relève nullement, selon l'auteur, de ce que les historiens appellent trop facilement la " réforme catholique " post-tridentine, mais traduit plutôt la volonté de Fregoso d'appliquer les préceptes d'un Briçonnet ou d'un Lefèvre, de faire vivre concrètement l'évangélisme français dans la péninsule. En des passages très intéressants et stimulants, l'auteur montre bien la complexité de la foi de Fregoso qui allie des élans réformateurs et des héritages évangéliques. Il n'est d'ailleurs pas le seul évêque à adopter une telle conduite dans la péninsule au cours des années 1530, puisque le nouveau pape Paul III s'allie alors les services du cardinal Contarini qui souhaite, dès 1535, réconcilier les catholiques avec le monde protestant. Dès lors, Fregoso est appelé à jouer un rôle majeur puisque Contarini aimerait l'avoir à ses côtés à Rome et le pape souhaiterait en faire un cardinal. Si le prélat refuse dans un premier temps ces deux propositions, 
préférant rester dans son diocèse, il est bien malgré lui contraint d'accepter le chapeau de cardinal en décembre 1539 et de se rendre à Rome (241). Mais, impuissant à imposer ses idées et fort isolé, notamment lors du concile de Ratisbonne où il est le seul à afficher des conceptions théologiques bien proches de celles des réformateurs allemands, il préfère bientôt quitter Rome. Après sa mort, qui survient le 22 juillet 1541, son nom est associé à celui de l'ennemi, il prend les traits de l'hérétique et ses œuvres sont mises à l'Index. Si le titre de l'ouvrage, «Condottiero, Cardinal, Eretico » prend alors tout son sens, il reflète surtout la complexité de ce courant évangélique de la première moitié du XVI siècle et la difficulté pour ses adeptes d'apparaitre comme des fidèles de Rome alors qu'ils souhaitent la conciliation avec l'ennemi protestant. La trajectoire humaine et spirituelle de Fregoso, qu'expose très bien G. Alonge, n'en finit pas d'interroger le lecteur sur ce milieu évangélique, son existence même, son influence et sa postérité. Afin de poursuivre la réflexion, il aurait été souhaitable que ce livre offre une bibliographie générale sur le sujet : l'appareil critique de l'ouvrage est en effet très réduit puisque seul un index des noms est proposé et aucune bibliographie, même brève, n'est présente. Cette absence regrettable ne nuit cependant pas au fait que ce très beau volume a le mérite de proposer une lecture fine et stimulante du parcours singulier d'un évangélique au temps du « Cinquecento ». Surtout, il invite son lecteur à poursuivre les recherches sur ce milieu évangélique définitivement si complexe et foisonnant.

MARIE BARRAL-BARON

Université de la Franche Comté

\section{Andreini, Isabella.}

Mirtilla, A Pastoral: A Bilingual Edition. Ed. Valeria Finucci, trans. Julia Kisacky.

The Other Voice in Early Modern Europe: The Toronto Series 62 / Medieval and Renaissance Texts and Studies 531. Toronto: Iter Press / Tempe: Arizona Center for Medieval and Renaissance Studies, 2018. Pp. xv, 291. ISBN 978-086698-588-8 (paperback) US\$49.95.

This bilingual edition of Isabella Andreini's 1588 play offers Italian and English texts side by side, as well as an extensive critical and annotated commentary that 